\title{
Amours et politique ; les politiques de l'Amour : à propos de Popi de Teodor ScorȚescu
}

Love and Politics; the Politics of Love: On Popi by Teodor ScorȚescu

Amor și politică; politicile amorului: Despre Popi de Teodor ScorȚescu

\section{Victor Ivanovici}

\section{OpenEdition}

Journals

Édition électronique

URL : https://journals.openedition.org/ceb/9608

DOI : $10.4000 /$ ceb. 9608

ISSN : 2261-4184

Éditeur

INALCO

Édition imprimée

ISBN : 978-2-85831-239-9

ISSN : 0290-7402

\section{Référence électronique}

Victor Ivanovici, « Amours et politique ; les politiques de l'Amour : à propos de Popi de

Teodor Scorţescu », Cahiers balkaniques [En ligne], 44 | 2016, mis en ligne le 30 novembre 2017, consulté le 07 juillet 2021. URL : http://journals.openedition.org/ceb/9608 ; DOI : https://doi.org/ $10.4000 /$ ceb.9608

Ce document a été généré automatiquement le 7 juillet 2021.

\section{(c) (1) 8)}

Cahiers balkaniques est mis à disposition selon les termes de la Licence Creative Commons Attribution - Pas d'Utilisation Commerciale 4.0 International. 


\title{
Amours et politique; les politiques de l'Amour : à propos de Popi de Teodor ScorȚescu
}

\author{
Love and Politics; the Politics of Love: On Popi by Teodor ScorȚescu \\ Amor și politică; politicile amorului: Despre Popi de Teodor ScorȚescu
}

Victor Ivanovici

1 Injustement oublié dans son pays natal, Teodor Scorţescu (1893-1976) appartient à l'époque de l'entre-deux-guerres de la littérature roumaine. Sans y occuper une position de premier rang, sa place reste très honorable parmi les figures de second plan.

2 Diplomate de carrière, ScorŢescu passa par plusieurs capitales européennes, dont Istanbul et Athènes qui lui laissèrent un fort penchant « orientaliste ». Vers la fin de la Seconde Guerre mondiale, il se trouvait en mission à Rome où, bien placé pour deviner quel sort serait réservé à la Roumanie dans l'après-guerre, il se garda de rentrer à la «base » lorsqu'il y fut rappelé. Après quoi il s'installa en Italie, d'autant plus qu'en 1930 il avait épousé la princesse Livia Borghèse. Ensuite, les informations précises sur son destin nous manquent presque complètement, on pourrait dire que Scorţescu se perdit dans l'exil.

3 Le reste n'est que suppositions et conjectures. Apparemment, le couple habita d'abord en Sicile, puis émigra en Argentine (à en juger d'après un dernier livre de l'auteur, paru en 1952, près de Buenos Aires, chez un petit éditeur de l'émigration roumaine). Revenu en Europe, l'écrivain passa, paraitt-il, le restant de sa vie dans le pays de sa femme.

4 Son activité littéraire avait commencé avec la poésie; par la suite, il aborda aussi le théâtre et la prose. Dans ce dernier genre, qui constituait sa véritable vocation, Teodor Scorţescu a laissé un roman et quelques nouvelles, dont Popi (1930), qui nous occupera ici, est sans doute la plus réussie.

5 À mon avis, la qualité maîtresse de cet ouvrage est un certain « air de famille ", non pas frappant, mais toutefois décelable à un niveau plus profond, qui fait penser aux fort 
connues Lettres persanes (1721), de Charles Louis de Secondat, baron de Montesquieu. De son temps, avec cet ouvrage, l'auteur gagna la faveur du public français en lui parlant de choses de chez lui, mais d'une voix étrangère. Certes, le retournement de l'exotisme allégorique n'avait rien de nouveau: comme presque tout dans la littérature, ce procédé ramenait à de multiples précédents. Et cependant, dans cette satire philosophique typique du XVIII ${ }^{\mathrm{e}}$ siècle, la simple convention romanesque finit par être homologuée comme une leçon de relativisme culturel. En effet, rien ne nous avertit mieux sur la vraie portée des valeurs propres que de les contempler avec les yeux d'autrui.

6 C'est à un exercice de ce genre que s'adonne Teodor ScorŢescu dans Popi, en nous proposant une histoire grecque racontée par un Roumain.

\section{Un « Persan » roumain en vacances dans la Grèce déchirée}

7 Le sous-titre de sa nouvelle, " 1922 », fait allusion à une date marquée de noir dans les annales de la Grèce moderne. Et pour cause : c'est l'année de la tristement fameuse "Grande Catastrophe d'Asie Mineure », ce désastre politique et humain qui mit un point final à la délirante "Grande Idée »-la chimère de la restauration de l'Empire byzantin - qui avait hanté tout le premier siècle d'existence de l'État grec. Dénouement d'autant plus douloureux que, pour un moment, la marotte irrédentiste semblait s'accomplir dans l'éphémère "Grèce des deux Continents et des cinq Mers " issue du traité de Sèvres pour être aussitôt anéantie par celui de Lausanne. Par contre, ce qui tint bon et même puisa une nouvelle vigueur dans la débâcle fut la «haine farouche qui déchirait la Grèce en deux ${ }^{1}$ ", depuis la Grande Guerre et sur le fond de la même Grande Idée. Les manuels d'histoire la rangent sous l'étiquette de la Discorde ou $\mathrm{du}$ "Schisme national », opposant les partisans du roi Constantin (deux fois déposé et deux fois rappelé sur le trône), à ceux du charismatique dirigeant libéral Elefthérios Venizélos.

8 Scorţescu nous rapporte l'essentiel de ce drame, dont le dernier acte se jouait ces jours-là : la défaite et la fuite des troupes grecques, l'évacuation de l'Asie Mineure, la révolte de l'armée humiliée et son débarquement à Laurion, l'éviction du roi Constantin, la chute du cabinet Gounaris, le procès et l'exécution expéditive « des Six ", tenus pour responsables du désastre... Cependant, dans la mesure où le narrateur de Popi contemple les faits d'un regard distancié, son récit devient une «lettre persane » (que même les Grecs d'aujourd'hui pourraient consulter avec profit).

Qui est derrière ce souriant regard étranger qui, en déplaçant les accents émotionnels, comme dans le «travail du rêve" freudien, crée un effet de distance que les autochtones n'étaient pas capables de prendre?

Ce regard appartient à un jeune cadre roumain en voyage d'affaires à Athènes ; pour lui, la conjoncture dramatique que la Grèce est en train de vivre ne représente qu'un simple inconvénient, car elle entrave le remboursement des crédits octroyés au gouvernement hellène par la banque Dacia Romană, qu'il représente en Grèce ${ }^{2}$. Sans trop le prendre à cœur (il paraît beaucoup plus ennuyé par l'impitoyable climat estival d'Athènes), il profite de ces vacances imprévues pour mieux connaître le pays et ses 
femmes. De cette option du narrateur-protagoniste découle le profil de genre de l'ouvrage : Popi sera donc une « étude de mœurs » et une histoire d'amour.

\section{Syndromes et manies grecques des « années folles »}

11 Le premier volet de ce diptyque s'inscrit dans la catégorie, très en vogue dernièrement, des recherches imagologiques. Non sans raison, car, de Popi, l'on peut tirer une image assez complexe de la Grèce et de ses habitants au début de la troisième décennie du siècle dernier, également connue sous le nom des « années folles ».

Dans cette image, le regard étranger, qui voit les choses du dehors, se juxtapose à la vision du dedans qu'ont les Grecs d'eux-mêmes, sans parler de leur propre regard vers l'extérieur, c'est-à-dire de l'idée qu'ils se font des autres. Il en résulte (pour ainsi dire) une stéréoscopie de stéréotypies. En premier lieu parce que toute imagologie travaille surtout avec des stéréotypes. En second lieu, parce que, dans ce genre d'études, tout dépend - pour citer un proverbe espagnol - del color del cristal con que se mira (" de la couleur de la vitre dans laquelle on regarde »). En l'occurrence, de l'« analyste » qui assume le traitement des stéréotypes. Celui-ci est le même narrateur dont on a déjà fait la connaissance ; les besoins du récit à la première personne le placent en position de "perceiver» (comme diraient les Anglo-Saxons), sans pour autant faire de lui un « raisonneur » (à la française). Au cœur de son époque frivole, il reste un touriste, par définition voué à la frivolité, mais son intelligence suffit pour qu'il en fasse bon usage.

De la hauteur de son manque de sérieux, il peut observer l'excès de gravité que les Grecs attachent à certains sujets. Tel est, par exemple, l'amour de la patrie qui, pour eux, est un absolu dépourvu de nuances et qui, parfois, devient un syndrome foudroyant. En grec moderne, cela s'appelle progonoplexie, et ses effets, de même que le mot, ressemblent franchement à l'apoplexie. Sans ambition de faire la phénoménologie de ce «mal d'ancêtres" (pourrait-on l'appeler aussi «aïeulite »?), notre «Persan » roumain décrit une large gamme de signes et de symptômes du syndrome patriotique.

À bord d'un navire en train de traverser l'Égée, une mer de dimensions plutôt modestes, le jeune Roumain surprend une dame athénienne dévorant la côte grecque d'un regard si avide qu'elle semble revenir du bout du monde. Le sympathique Levantin Spyros Valassinos, qui se dit descendant des «comtes de Valassin » et qui possède un passeport français (grâce auquel il a échappé de justesse au massacre de Smyrne), se félicite de ce que le sang grec se soit entièrement substitué dans ses veines, à celui des Croisés.

Les Grecs d'aujourd'hui "parlent de l'ancienne Hellade comme si c'était hier », et traitent ses " grands défunts comme leurs grands-pères personnels » (p. 34-35). Issus de la race qui est « le blason de la planète » - et par cela, se fichant des titres de noblesse -, ils ennoblissent le monde entier de leurs grands hommes : « N'est-ce pas nous qui avons donné à la Russie un Capo d'Istria ? ", " N'est-ce pas nous qui avons donné à la France un Napoléon ?», l'histoire de Roumanie « n'est-elle pas pleine de noms grecs?» (p. 36).

L'humanité entière leur devant gratitude, quoi de plus naturel que de considérer les autres en fonction de leur attitude envers la Grèce ? Curieusement, ils n'en veulent pas trop aux Turcs: sans doute parce que chaque faction grecque impute la défaite en Asie Mineure à la faction opposée. Les Français sont considérés comme presque aussi 
coupables, car ce sont eux qui ont armé les kémalistes. La Roumanie, pays voisin, mais sans frontières communes et, de ce fait, sans problèmes territoriaux ou de minorités avec la Grèce, est convoitée en tant que «terre de l'abondance et des délices, une espèce d'Eldorado pour les Balkans » (p. 23). Beaucoup de Grecs y ont vécu et y vivent encore, les royalistes l'aiment du fait qu'elle est la patrie de l'épouse du Diadoque et presque tous, quoi qu'en pensent les Roumains, la voient comme une sorte de « colonie grecque » (p. 36).

Et les exemples peuvent continuer.

Il n'est pas difficile de deviner de quel sourire le protagoniste contemple ce genre de "couleur locale»: amusé, condescendant, souvent ironique ou même cynique, ce sourire n'est jamais arrogant. Notre "Persan" a beau se moquer des idées fixes des autochtones, il ne cesse de regarder les Grecs avec une grande sympathie. Comme tout inconditionnel, il les aime non pas en dépit de leurs failles, mais pour elles. Même dans les occasions où ils arrivent à l'agacer, en le faisant sortir de sa réserve polie. Par exemple quand il dénonce devant ses interlocuteurs la folie qui ravage leur pays :

Vous êtes [...] le peuple le plus intelligent que je connaisse. Lorsque vous traitez des affaires, votre vision du monde s'ajuste à la réalité comme un gant. Où que vous alliez, vous êtes les maîtres de la situation. Les gens, ainsi que les lieux, sont chez vous aussi beaux qu'il y a trois mille ans. Vous pourriez être heureux. Mais, de haut en bas, vous êtes tous empoisonnés par la politique (p. 78).

Peine perdue, car qui connaît les Grecs sait qu'ils adorent leurs défauts : « Nous avons horreur de l'équilibre » (loc. cit.), lui réplique-t-on fièrement, puis on lui fait savoir que métabolé - le terme pour désigner le changement ou l'évolution - signifie littéralement « jeter par-dessus », c'est-à-dire " larguer » qui ou quoi que ce soit. Quoique douteuse philologiquement, ladite interprétation nous suggère que, pour les Grecs, le progrès n'est point une croissance faite de lentes accumulations, mais plutôt d'expulsions et d'exclusions violentes. C'est ce qui est en train de se passer à l'époque de la grande Discorde où, avec un acharnement inouï, la Grèce vénizéliste s'apprête à « larguer » la Grèce royaliste et vice-versa.

Au niveau du quotidien, ce rejet revêt des aspects «folkloriques ». Gare à vous, par exemple, si vous commandez un ouzo dans le mauvais café : cette boisson est l'apéritif préféré du roi Constantin! Surtout, n'essayez pas de mettre en contact deux Grecs sans que l'un d'eux ne vous le demande : on ne sait jamais à quelle faction ils appartiennent ! Des proches parents se croisent sans un regard, faisant semblant de s'ignorer. L'enfance est plus franche : une fillette de six ans enfonce ses ongles dans la chair de sa cousine, venue lui annoncer la victoire électorale des adversaires. Même l'amour n'échappe pas à l'emprise de la Discorde.

21 Pour achever cette analyse de Popi en tant qu'« étude de mœurs", il faut encore signaler que l'auteur n'omet pas d'enregistrer aussi la truculence de ces «années folles ", leur côté sinistre faisant pendant à l'image souriante qu'il préfère nous donner de la Grèce. Il s'agit de la véhémence cannibale qu'atteignent dans ce pays les passions politiques. Ainsi, les révolutionnaires au pouvoir, non contents de "larguer» le monarque du trône, s'arrangent aussi pour « larguer » ses ministres... au poteau. Après quoi les gamins athéniens marchandent dans la rue des petits morceaux de crânes, ramassés à l'endroit de l'exécution. 


\section{La Grèce au féminin}

Comme annoncé, un autre point de vue à partir duquel on peut aborder la nouvelle de Scorţescu est celui de l'histoire d'amour. Cet aspect n'est qu'un détail du tableau de mœurs que nous offre l'ouvrage, puisque l'idylle du protagoniste avec une jeune athénienne s'inscrit dans le contexte de son intérêt soutenu pour la Grèce au féminin.

Comme dans les sciences naturelles, tout commence par des observations empiriques. D'abord sur un spécimen choisi au hasard: Mme Soliadès, par exemple, rencontrée par le Roumain pendant son voyage en bateau, qui « portait son pays sur elle de façon plus frappante que si elle allait vêtue en costume national. Son front, son nez, ses lèvres, son profil net s'obstinaient à faire publicité à une beauté désuète depuis trois mille ans " (p. 20). Puis le champ de recherche s'étend sur un échantillon plus large de la même espèce, dont le comportement collectif est surpris dans son milieu naturel, la terrasse d'une confiserie en pleine animation estivale :

Des yeux en amande jetant par les faces-à-main des regards incitants, abondance de nez classiques ennoblissant les profils, des figures basanées aux nuances olivâtres entre lesquelles brillait, ça et là, le teint d'ambre des Smyrniotes blondes, de jolies têtes se penchant l'une vers l'autre, parlant en même temps, des bras nus agitant des éventails, des robes vaporeuses, décolletées, tout cela jouait devant mes yeux, dans un vacarme bouillant de perroquets en délire (p. 31).

Par la suite, la teneur de la démarche se modifie: de ce genre d' "ornithologie» empirique, le chercheur passe à une «étude de cas » à la manière des sciences sociales. La raison en est la connaissance de Popi, en qui le Roumain trouve une informatrice de premier ordre et, tout à la fois, un objet idoine d'investigation. Dorénavant, la recherche se personnalise, s'érotise même, et la physiologie de la femme grecque tourne peu à peu au récit libertin.

\section{Suivons les phases de cette évolution.}

Popi représente dignement la beauté locale, bien que sa physionomie ne soit pas des plus classiques. Elle a « un teint mat, des yeux en amande, ombragés par des cils fléchis vers le haut; sous son nez naif, un peu retroussé, une bouche rouge aux lèvres pulpeuses » (p. 25). Son corps est aussi désirable que le visage :

La jambe, légèrement cambrée, finissait par un genou arrondi comme une pomme, que la robe courte laissait à découvert. Les hanches amples, courbées avec la grâce d'une lyre [...], etc. (p. 64).

Sa voix est « claire et musicale » et « le typique zézaiement des Grecs quand ils parlent une langue étrangère lui prête une grâce enfantine » (p. 26), lorsque, dans un français bien maitrisé, elle fustige la félonie de la France. La même voix, mise à chanter, devient " chaude et vaste ", révélant peu à peu, «tel un corps qui se déshabille, une sensualité vibrante » (p. 52).

La jeune femme est «veuve », en l'occurrence « veuve de vivant " (zontochera), comme l'on appelle en Grèce les divorcées. Pour cette raison, elle est retombée sous la férule de sa famille et, à 23 ans, elle doit cacher ses amourettes des yeux sévères de l'« oncle Thémistocle " ainsi que des voisines vouées aux commérages. Si un moment elle se montre complaisante envers un homme, elle tient absolument à ce que celui-ci n'en tire pas de «fausses conclusions ». " Madame, vous parlez comme une Roumaine » (p. 33), réplique avec sincérité son compagnon, se souvenant peut-être d'une certaine histoire 
drôle, selon laquelle ses concitoyennes ont l'habitude de demander, après: «Et maintenant que vas-tu penser de moi ?»

Avec la même sympathie et le brin d'ironie dont il regarde les choses du pays, le narrateur rapporte les ruses et les efforts que Popi met en œuvre pour s' « émanciper ». En commençant, bien sûr, par la diète. Puisque les Grecques, dès qu'elles " attachent leur âne "(dit le peuple), c'est-à-dire une fois mariées, commencent à prendre du poids : "C'est ça le cachet de la félicité domestique»(p. 50). En revanche, Popi est sortie mince de cet obèse paradis, et entend le rester ; le prix en est de ne dîner « que des oranges [...] et une petite bouteille de Rezinato » (p. 49). De surcroît, elle fait du skating et va souvent nager (sur des plages séparées pour les hommes et les femmes). Enfin, dans une Athènes qui n'est encore qu'un grand village, elle s'arrange pour rencontrer (comme par hasard!) qui elle veut.

En politique surtout, elle a ses propres convictions et les défend, avec courage et franchise, contre qui que ce soit. D'ailleurs, dans ce pays où les mâles font la loi, la Discorde devient plus farouche lorsqu'elle prend un visage féminin. Ainsi, Mme Soliadès ne mâche pas ses mots pour traiter son cousin royaliste de "dégénéré " (p. 32). Quant à Popi, elle n'hésite pas non plus à s'afficher, en plein territoire ennemi (un café fréquenté par les vénizélistes), comme zélatrice de Constantin, ni à affronter, pour la même raison, son frère aîné de retour de la guerre.

31 Si la politique est un terrain acquis aux femmes, que se passe-t-il dans le domaine auquel on pense par excellence lorsqu'on parle d'émancipation? Qu'en est-il du libre exercice de la sexualité féminine? Les choses y sont plus compliquées qu'ailleurs en raison de l'influence de plusieurs paramètres, dont quelques-uns sont extérieurs (parmi eux, la politique elle-même), d'autres intérieurs ou "intériorisés » (telles la psychologie, les mœurs, les mentalités). Et pour aller du côté « libertin» de l'histoire, c'est de tout ce complexe que doit tenir compte l'aspirant séducteur.

La femme grecque suit en amour un scénario soi-disant romantique (arrivé dans les Balkans, à l'instar du skating, lorsque sa vogue était déjà passée en Occident). Foncièrement mensonger - à en croire René Girard (Mensonge romantique et vérité romanesque, 1961) -, ce scénario n'est qu'un succédané du sexe et fait de ses pratiquantes, qu'elles le veuillent ou non, des «allumeuses ». Popi, qui se dit «trop sentimentale» et qui veut «être aimée» passionnément (c'est pourquoi elle a abandonné un mari plutôt tiède), comble sa solitude avec l'amitié d'une... chèvre. En rêvassant à l'amant idéal, elle soumet ses amoureux réels à des ordalies meurtrières. Notre "Persan", par exemple, manque de peu de mourir d'insolation à l'heure la plus brûlante de la journée à cause du rendez-vous qu'elle ne lui donne que pour se faire voir à la fenêtre.

Il ne s'agit pas de sadisme, mais de la règle d'un jeu qui exige que la femme tombe dans les bras du soupirant juste à la fin, "tardive, comme une récompense nationale " (p.60). Et celle qui joue ce jeu n'est pas de glace; elle a un corps qui (comme dirait le poète) « laisse percer des obscures paroles » : un regard " où j'aurais navigué toute la nuit » (p.51), une bouche qui, sous les baisers, a "la dure fraîcheur d'une prune » (p. 54), des seins qui, sous l'empire de l'émotion, se mettent à «frémir rythmiquement» (p. 81). Mais, pour que ces appels équivoques se convertissent en messages clairs et que leur récepteur en devienne le destinataire, il lui faut un moyen prodigieux, qui (comme dans un roman de Diderot) parvienne à faire parler le «bijou » trop discret de sa bien-aimée. 

scénario romantique : « Pour Popi, notre idylle devait absolument passer en ce moment par une forme musicale, afin qu'elle accomplît les exigences d'un rituel consacré » (p. 53) ; en même temps, la musique représente l'épreuve suprême d'amour, après laquelle la récompense est en vue. En l'écoutant chanter, le Roumain a l'intuition de son tempérament fort érotique, et c'est en rêvassant sur une mélodie orientale qu'il parvient à l'imaginer complètement nue, ondoyant de son ventre et fredonnant l'inévitable 0-po-po.

Malheureusement, la ressource musicale reste hors de la portée du soupirant, car - ô fatalité ! - la voix lui manque. Par force, il doit ou bien se résigner au mutisme du «bijou » convoité, ou bien le réveiller par une astuce. Évidemment, il choisira la deuxième solution, moyennant les services d'un chanteur à gages. Cette ruse est en train de produire ses effets, jusqu'au moment où le Cyrano d'occasion se rebelle contre son commanditaire, voulant s'emparer, lui, du « bijou » de Popi.

Tout paraît donc perdu. Et pourtant le menteur démasqué peut encore « accoucher » la vérité à la manière de Socrate. Par ses propos, il fait en sorte que les deux Grecs révèlent leurs loyautés contraires. Ainsi, il tire parti d'une passion qui, en principe, n'a rien d'érotique, mais qui, au temps de la Discorde, exerce une forte influence sur les « bijoux » locaux. La scène finale de Popi abrite ce coup de théâtre, d'autant plus génial qu'il est improvisé sur place :

Je regardais autour de moi, cherchant une sortie honorable. Soudainement, je tressaillis. Mes yeux tombèrent sur un petit portrait du roi Constantin, suspendu au mur près de la porte. Une idée me vint à l'esprit.

- Je dois le reconnaître, dis-je en pointant Spyros de mon doigt, ce fervent royaliste s'est avéré plus fort que moi!

Vexé, Spyros bondit comme mordu par une vipère :

- Royaliste, moi ?

Puis il sourit et, en penchant sa tête en arrière, cria à pleins poumons :

- Vive Venizélos! Toute la Grèce est avec lui !

Popi, affolée, leva son front. Des pareils propos sacrilèges sous son toit!

- La Grèce est fidèle au roi Constantin! hurla-t-elle d'une voix suffoquée par la rage (p. 81).

Après quoi le rival est chassé, le Roumain reste le maître du champ de bataille et le «bijou » convoité revient en trophée au vainqueur.

\section{L'impossible (et inévitable) triangulation du désir}

Dans les termes d'Aristote, ce final constitue une péripétie, c'est-à-dire le «renversement» (comique) du statu quo ante. Pour mentionner encore une fois l'ouvrage déjà classique de René Girard, le renversement en question met à l'échec un scénario fautif, le "mensonge romantique», au profit de la "vérité romanesque ". Celle-ci nous apprend que nos désirs ne sont presque jamais autonomes : nous désirons par mimétisme, ce que désire un tiers, le "médiateur». Quand ce dernier se trouve socialement hors de la portée du sujet, voire hors du monde réel, la médiation « extérieure » est sublimée sous forme d'un idéal inatteignable, mais, pour ainsi dire, stimulant. Par contre, quand le médiateur est réel et au même niveau que le sujet, il se transforme en rival et en obstacle pour l'appropriation de l'objet désiré. De ce désir au second degré découle le «triangle amoureux », la plus solide figure du récit occidental. 
Évidemment, la nouvelle de ScorŢescu s'appuie sur un tel triangle qui justement parce qu'il est stable, en géométrie comme en littérature, admet à ses sommets des « rôles " changeants.

J'y vois au moins trois variantes :

- l'une, assez simple, n'est que le triangle «classique »: désireur/désirée/médiateur (interne); en l'occurrence : Spyros - Popi - le narrateur. Le troisième suscite le désir chez le premier, ou le premier l'imite ; ce qui revient à la même chose, dès lors que la désirée reste Popi. Se révoltant contre le médiateur, le «Cyrano » mimétique ne vise pas à s'affranchir de lui, mais à usurper sa position près de l'objet commun de leur désir ;

- la seconde variante est plus compliquée du fait que la médiation, externe, y est politique dans sa forme et psychanalytique dans son fond ${ }^{3}$. Suivant les injonctions d'une double figure paternelle (Venizélos et le roi Constantin), Spyros et Popi désirent, sont désirés - l'un(e) l'autre, l'un(e) par l'autre - et finalement renoncent au désir, à cause de l'extériorité du/des médiateur(s). La versatilité des rôles est extrême, l'autonomie du choix presque nulle: comme Don Quichotte dans ses rapports avec Amadis de Gaule, ils laissent choisir pour eux leurs deux «pères » politiques (occupant le troisième sommet du triangle). Ceux-ci étant incompatibles politiquement, ceux-là seront incompatibles du point de vue érotique. Par conséquent, Spyros est expulsé du triangle et la place restée vide est comblée par son rival ;

- dans cette dernière variante, la posture du Roumain n'est guère commode. Que fera-t-il afin que son désir rentre dans le schéma triangulaire? Qui en sera le médiateur (externe, car, de l'interne, il n'est plus question)? Un roi, qu'il dit aimer juste pour faire plaisir à la dame, mais qui, au fond, reste non seulement hors de son monde, mais aussi de son intérêt? Ou bien l'amour qu'elle voue au roi par complexe d'Électre, qui est, disons, l'œdipe au féminin et qui, comme tout complexe, crée plutôt des complications?

41 Pour une fois, la politique vient le sortir de ces dilemmes. En bannissant le monarque et en tuant ses vicaires, les Grecs donnent une solution à l'impossible (et à la fois inévitable) triangulation du désir. Lorsque du père-médiateur ne restent que quelques osselets, rien n'empêche plus Popi de se livrer à son amant.

Si elle le fait en orpheline, la mort dans l'âme et avec ses lèvres scellées, son «bijou » est enfin éloquent :

Quelques instants le corps de Popi flotta dans les eaux grises du néant, puis, peu à peu, elle revint à la surface. Un frisson contracta ses jambes nues... Elle gémit doucement et, comme dans un rêve, leva ses bras et les noua autour de mon cou. Je me penchai sur elle jusqu'à sentir son haleine chatouillant mon visage.

Sous mon baiser, ses lèvres étaient froides comme la bouche d'une statue (p. 83).

\section{NOTES}

1. Teodor SCORŢESCU, Popi, in Concina prădată, édition par Ion SimuŢ, Cluj-Napoca: Dacia/ „Restituiri”, 1982, p. 21 [nous traduisons]. Les références au texte sont désormais données dans le corps du développement.

2. Cela nous rappelle quelque chose, n'est-ce pas? 
3. N'oublions pas que la psychanalyse, avec ses pulsions et ses complexes, n'est qu'un cas particulier du « mimétisme » selon René Girard.

\section{RÉSUMÉS}

Dans ces pages, je me propose d'analyser la nouvelle Popi (1930) de Teodor ScorŢescu (1893-1976), auteur injustement oublié dans son pays, dans le but de déceler les leçons de relativisme culturel qui se dégagent d'une histoire à sujet grec racontée par un Roumain. L'ouvrage nous propose une intrigue amoureuse se déroulant sur le fond de la grande «Discorde nationale "

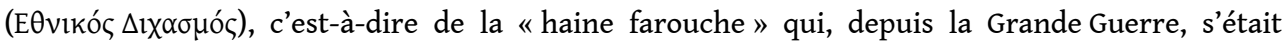
emparée de la Grèce, la déchirant entre les partisans du roi Constantin et ceux du dirigeant libéral Elefthérios Venizélos. Un jeune Roumain, en voyage d'affaires dans ce pays humilié par la défaite et secoué par des convulsions révolutionnaires, observe les passions politiques effrénées de ses hôtes, d'abord avec un détachement amusé, puis en apprenant à les mettre à profit pour séduire une belle Athénienne et pour éloigner d'elle un rival fâcheux. La double posture du héros narrateur confère à la nouvelle de ScorŢescu un double profil de genre. D'un côté, il s'agit d'une " étude de mœurs ", susceptible d'enseigner aux Grecs les avantages à contempler les choses de chez eux avec les yeux d'autrui, et, en ce sens, qui ressemble, toutes proportions gardées, à une «lettre persane » à la Montesquieu. De l'autre côté, Popi est aussi une histoire « libertine » qui, avec un petit clin d'œil à Diderot, porte sur l'art de faire parler un « bijou » féminin trop discret.

In this paper, I would like to analyze the nouvelle Popi (1930) by Teodor ScorŢescu (1893-1976), an author unjustly forgotten in his country, in order to identify cultural relativism lessons emerging from a story with Greek subject told by a Romanian. The narrative relates a love affair on the

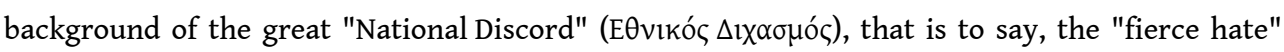
which, since the World War I, had torn Greece, between supporters of King Constantine and those of the liberal leader Eleftherios Venizelos. A young Romanian, on business trip in this humiliated by defeat and shaken by revolutionary convulsions country, observes the raging political passions of his hosts, first with amused detachment, and then he learns to put it to use to seduce a beautiful Athenian woman and to drive away an annoying rival. The double posture of the narrator gives to Scorţescu's story a double kind of profile. On the one hand, as a "study of manners", it may teach the Greeks the benefits to contemplate domestic things with the eyes of a stranger, and in this sense seems, relatively speaking, a "Persian letter" à la Montesquieu. On the other hand, Popi is also a "libertine" story that, with a little nod to Diderot, focuses on the art of make speaking a too quiet feminine "jewel".

În paginile de faŢă, îmi propun să analizez nuvela Popi (1930) de Teodor Scorţescu (1893-1976), autor pe nedrept uitat în propria-i Țară, în scopul de a pune în evidenţă lecţiile de relativism cultural ce se desprind din această istorie cu subiect grecesc relatată de un român. Nuvela ne propune o intrigă amoroasă, desfăşurată pe fondul marii „Discordii NaŢionale”

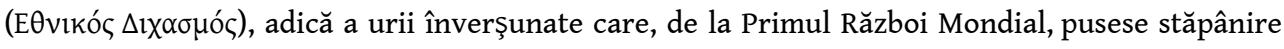
pe Grecia, sfâşiind-o în două tabere: partizanii regelui Constantin şi aceia ai liderului liberal Eleftherios Venizelos. Un tânăr român, în voiaj de afaceri în această Ţară umilită de înfrângerea militară și zguduită de convulsii revolựionare, observă patimile politice dezlănŢuite ale gazdelor, mai întâi cu o detaşare amuzată, iar mai apoi învaţă să profite de ele pentru a seduce o 
frumoasă ateniană şi pentru a îndepărta de ea un rival sâcâitor. Dubla postură a eroului narator conferă povestirii lui ScorŢescu un dublu profil de gen. Pe de-o parte, avem de-a face cu un „studiu de moravuri”, susceptibil de a-i învăŢa pe greci să contemple afacerile lor domestice cu ochii străinului, şi în acest sens seamănă, păstrând proporŢiile, cu o „scrisoare persană” à la Montesquieu. Pe de altă parte, Popi este și o istorie „libertină” care, făcând uşor cu ochiul lui Diderot, dezvoltă arta de a face să vorbească un „bijou” feminin prea discret.

\section{INDEX}

Index géographique : Roumanie

Mots-clés : royalistes, royalistes, Venizélos Elefthérios (1864-1936), ScorŢescu Teodor

(1893-1976), ScorŢescu Teodor (1893-1976), Venizélos Elefthérios (1864-1936), « bijou » féminin, «bijou » féminin

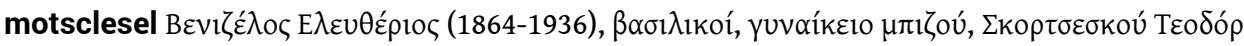

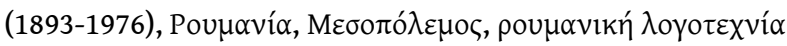

Keywords : Royalists, Feminine "Jewel", Venizelos Eleftherios (1864-1936), ScorŢescu Teodor (1893-1976), Romania, Interwar period, Romanian literature

Thèmes : Littérature roumaine

motsclesmk РОМАНИЈА, ПЕРИОД МЕЖДУ ДВЕТЕ СВЕТОВНИ ВОЙНИ, РОМАНСКА КНИЖЕВНОСТ, ЖЕНСКИ НАКИТ

motsclestr Venizelos Elefterios (1864-1936), Scorţescu Teodor (1893-1976), Kralcılar, Kadınsı mücevher, Romanya, İki savaş arası dönem, Romen edebiyatı

Index chronologique : entre-deux-guerres (1918-1939)

\section{AUTEUR}

\section{VICTOR IVANOVICI}

Université de Thessalonique 